\title{
Data-Driven Model-Free Control of Torque-Applying System for a Mechanically Closed-Loop Test Rig Using Neural Networks
}

\author{
Aida Parvaresh ${ }^{1}$ - Mohsen Mardani2,* \\ ${ }^{1}$ K.N. Toosi University of Technology, Iran \\ ${ }^{2} \mathrm{ACECR}$, Sharif University of Technology Branch, Iran
}

This paper presents a data-driven approach that utilizes the gathered experimental data to model and control a test rig constructed for the high-powered gearboxes. For simulating a wide variety of operational conditions, the test rig should be capable of providing different speeds and torques; this is possible using a torque-applying system. For this purpose, Electro-Hydraulic Actuators (EHAs) are used. Since applying accurate torque is a crucial demand as it affects the performance evaluation of the gearboxes, precise modelling of the actuation system along with a high-performance controller are required. In order to eliminate the need for to solve complex nonlinear equations of EHA that originate from friction, varying properties of flow and similar, a data-driven system based on neural networks is used for modelling. In this manner, the model of the system, which captures the whole dynamic of the system, can be obtained without any simplifying assumptions. The model is validated with experimental data, and the learning factors are set to zero to reduce the high computational costs. After that, another network of neurons is used as a controller. The performance of the proposed controller under normal conditions and in the presence of disturbances are investigated. The results show a good tracking of this controller for various reference inputs in different conditions with acceptable characteristics. Additionally, the obtained results are compared with a conventional proportional-integral-derivative (PID) controller results, and the superior features of the proposed scheme is concluded.

Keywords: identification; data-driven system; closed-loop test rig; hydraulic actuator; neural networks

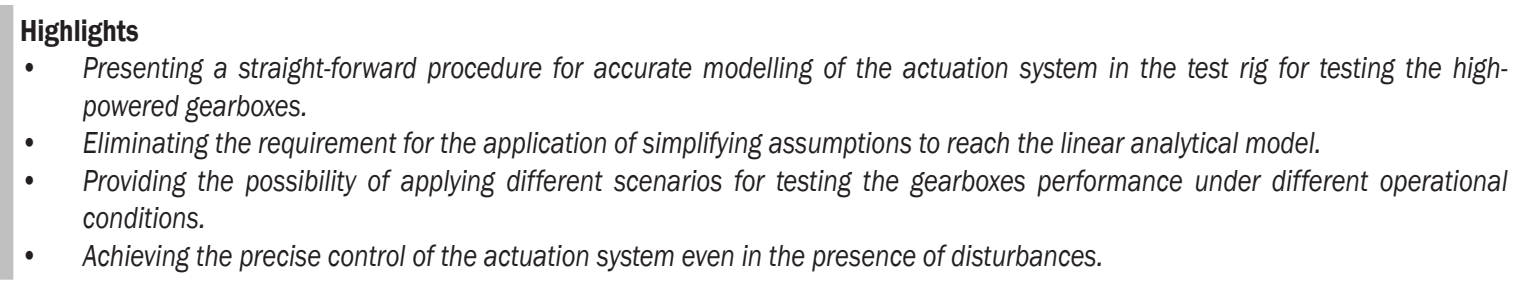

\section{INTRODUCTION}

Ensuring safety and performance along with the reduced maintenance costs of high-powered gearboxes, which are mostly used in helicopters and wind turbines, are of great importance in industrial applications. Since in-field testing of such equipment is often impossible, time-consuming, and not costefficient, test rigs are developed [1] and [2], for primary assessment of the capability and performance of the components in industrial use. Full-sized test rigs can provide realistic conditions so that the performance and operation of the systems can be evaluated. These test rigs can be designed both in closed-loop or openloop schemes. Test rigs operating based on the closedloop principle can reduce energy consumption up to $95 \%$ compared to open-loop schemes, so they are superior in testing high-powered gearboxes when a great amount of energy is required.

Different types of test rigs have been developed to investigate different features based on desired parameters to be tested. In a study by Åkerblom [3], a closed-loop recirculating power test rig was developed for testing gearboxes under controlled environments. Different parameters, including noise, gearbox life, and efficiency, were studied in a gearbox system consisting of two similar gearboxes. In the studied system, one gearbox was tilted using a hydraulic cylinder. In [4], Arun et al. reviewed different test rigs; they also fabricated a new test rig. A novel test rig was proposed by Mihailidis and Nerantzis [5], which was consisted of a planetary gear box for applying variable torques and speeds. Palermo et al. [6] constructed a precise closed-loop test rig to evaluate the dynamic behaviour of gear pairs in different operational conditions. Their fabricated test rig consisted of a test side to test the desired gearbox and reaction side to close the power train. Mozafari et al. [7], represented the preliminary, conceptual, and detailed design of a test rig that was developed for high-powered gearboxes. Their rig was a closed-loop mechanical type and used hydraulic torque applying system for providing the desired torque. In order to reach the desired position of the hydraulic actuators, they implemented on-off and proportional-integral- 
derivative (PID) controllers [8] as well as fractional order PID [9].

For conducting dynamic tests in high-powered gearboxes and for actuating the system, the torque should be applied automatically employing a torqueapplying system. These systems should be able to provide variable torques and speeds to simulate the operational conditions of high-powered transmissions. Different systems can be used for this purpose, among which, hydraulic systems would be the best choice due to their superior features. Compared to the other equipment for applying torque, electrohydraulic actuators (EHAs) can provide higher power-to-weight ratios considering the volume limitations, higher forces, reduced size of equipment as well as the robustness improvement. In addition, they are inherently more stiff and rigid so that the precise position control can be achieved. Also, they provide faster response, which is a desirable feature in industrial applications [10] and [11]. However, despite the noticeable advantages of these systems, their dynamic behaviour is highly nonlinear due to the nonlinear characteristics of flow and pressure, including varying bulk modulus, compressibility, and viscosity [12]. So, prior to the control of these systems, an accurate model, which represents the complete dynamic behaviour of the system should be developed. Despite the extensive published studies, a lack of precise and appropriate modelling is observed. Different methods have been utilized to overcome these problems. Some researchers attempt to overcome this problem via the linearization of equations and using linear control procedures to control these systems. However, by the use of linear control strategy, some portion of the dynamic system behaviour would be lost during the linearization of the system. Some researchers also used simplifying assumptions, such as neglecting leakage or flow compressibility. The obtained model by these approaches would be valid just in the adjacent of the linearization point. Additionally, the use of linear controllers would not lead to high-performance control. In some other researches, higher-order linear models were used to design controllers. However, these models also suffer from the validity problem in other points rather than operating points. In [13], a method for parameter identification of nonlinear terms in the model of the system was proposed. It was observed that considering the identified nonlinear effects, increases the accuracy of the model, significantly. In addition, in another study [14], the identification of the nonlinear effects, including friction coefficient was conducted using the input-output data.
The control of EHAs and developing an appropriate controller to satisfy the desired requirements are of great importance regarding their various applications. The control scheme can be designed either in a force control mode or displacement control mode. Considering the easier implementation of displacement control mode from the practical point of view, better performance as well as disturbance rejection characteristics, this mode is widely used in controlling EHAs [15]. Many nonlinear adaptive control approaches were used for controlling EHA, among them, the usage of sliding mode controller [16], back-stepping [17] and [18], feedback linearization controller [19] and [20] and so on can be mentioned. Detiček and Župerl [21], presented a novel hybrid-fuzzy control scheme for positioning the EHA in practical applications. Nonlinear control schemes are rarely utilized in industrial applications due to their need for a mathematical model as well as the complexity of tuning the parameters. Achieving high accuracies in the position tracking of hydraulic actuators are of great importance; however, parametric uncertainties, as well as nonlinearities, are the major problems in this field [22]. Parametric uncertainties can be compensated by the use of adaptive control schemes, while nonlinearities can be handled by robust controllers. To take advantage of these two schemes simultaneously, intelligent controllers are proposed. Guan and Pan [18] presented a nonlinear adaptive robust control procedure with unknown parameters for an EHA, by combining a back-stepping technique and a simple robust control. Haung et al. [17] used an incremental nonlinear dynamic inversion control technique for controlling a hydraulic actuator in the presence of system uncertainties. They implemented their proposed approach on a 6-degree-of-freedom (DOF) hexapod hydraulic robot.

In industrial applications, sometimes the use of popular theoretical control methods, which represents superior performance, is not possible due to the occurrence of some unpredicted and unexpected effects [23]. Model-free controllers, which do not require an explicit model of the plant, are the best choice in these applications, such as classic PID controllers. In these types of controllers, high and intensive modelling work are not required. Among the advantages of model-free control schemes, easy implementation and tuning of the coefficients can be mentioned. The model-free procedure provides good control features without considering an accurate model of the system [24]. These models aim to approximate the system dynamics using the gathered information from the embedded sensors that are 
estimated and updated in each time samples. It is evident that through using this control scheme, we can overcome the difficulties in mathematical modelling and improve the practical implementation.

Some model-free control schemes were used for controlling EHA. Neural networks (NNs) are universal approximators, which means that they can be used as a black-box estimator applicable for the systems with parametric uncertainties and nonlinearities [25]. Tremendous interest has been devoted to NNs, according to their outstanding performances in learning, adaptation, generalization, optimization, and control [26]. From the theoretical point of view, a continuous function can be approximated to a desired accuracy, which enables the modelling of the complex nonlinear system using NNs. In many research studies, NNs are used for control and modelling [27]. These structures are used to learn the features and characteristics of the system, which can be used as the model, instead of obtaining the explicit dynamic of the system. According to some studies, these controllers can be classified as model-free controllers as they do not use the exact model of the system. The existing complexities in the control problem of industrial systems make the neural network approach as a popular method. Learning the control scheme using NNs was proposed for enhancing the trajectory tracking performance. Yao et al. [25] developed an advanced nonlinear controller for the hydraulic system to obtain position tracking in the presence of various disturbances. They used a neural network estimator to improve the disturbance compensation. In general, using these networks have significant advantages over other controllers. In this paper, we aim to take the advantages of NNs for modelling and controlling of a hydraulically-actuated test rig.

The rest of the paper is organized as follows: In Section II, a brief introduction of the fabricated test rig, which is a mechanically closed-loop test rig with a hydraulically-driven torque applying system, is presented. Then, in Section III, the data acquisition procedure required for modelling of the system is explained. After that, in Section IV, the proposed algorithm for modelling and controlling the actuation system using neural networks is described. Then, the results of neural-network modelling and controlling for different conditions are presented in Section V. Finally, Section VI is dedicated to the conclusions derived from this research.

\section{SYSTEM DEFINITION}

The studied test rig is a mechanically closed-loop test rig that was designed and fabricated in Sharif University of Technology branch of Academic Centre of Education, Culture and Research (ACECR). The mentioned test rig was designed so that it has low energy loss; it also provides wide ranges of torques and speeds. Therefore, it is appropriate for testing the high-powered gearboxes that are commonly used in aeronautic industries. The specification of the test rig and torque-applying system are provided in Table 1. In Fig. 1, the schematic and real test rig are depicted. To apply the required torque to the testing system, a torque-applying system that consists of a planetary gearbox is used. By rotating the ring of this planetary gearbox, the system is rotated. The rotation of the ring of this planetary gearbox is provided by the linear hydraulic actuators, which are depicted in Fig. 2. It is noteworthy that all criteria, including stresses, strains, safety fractures, and failure and similar, are provided in the design procedure [7]. For sensing the displacement of the actuator rod, two displacement sensors are utilized with the maximum measurement course of $75 \mathrm{~mm}$, which is larger than the course length of the actuator. A hydraulic circuit is designed for controlling the hydraulic actuator; in addition, a $4 / 3$ directional valve is used for changing the direction of the hydraulic actuator. The actuation system consists of a three-phase electrical motor, a positive displacement pump, a container, a safety valve, and a pressure indicator. More information about this test rig and actuation is provided in [28].

Table 1. Specifications of the test rig and torque applying system

\begin{tabular}{llc}
\hline & Specification & Value \\
\hline \multirow{2}{*}{ Test Rig } & Max loading capacity & $365 \mathrm{~kW}$ \\
\cline { 2 - 3 } & Max rotational speed & $3000 \mathrm{rpm}$ \\
\hline \multirow{3}{*}{$\begin{array}{l}\text { Torque applying } \\
\text { system }\end{array}$} & Max hydraulic actuator course & $60 \mathrm{~mm}$ \\
\cline { 2 - 3 } & Max required force & $10 \mathrm{kN}$ \\
\cline { 2 - 3 } & Force applying arm & $175 \mathrm{~mm}$ \\
\cline { 2 - 3 } & Max rotation & $20 \mathrm{deg}$ \\
\hline
\end{tabular}

\section{DATA ACQUISITION}

The model obtained from the neural network structure does not require any mathematical relationships. It is a data-driven system, which uses input and output data of the system for training the desired network. Therefore, gathering data is an important step for modelling. The data collection procedure is depicted in Fig. 3. 


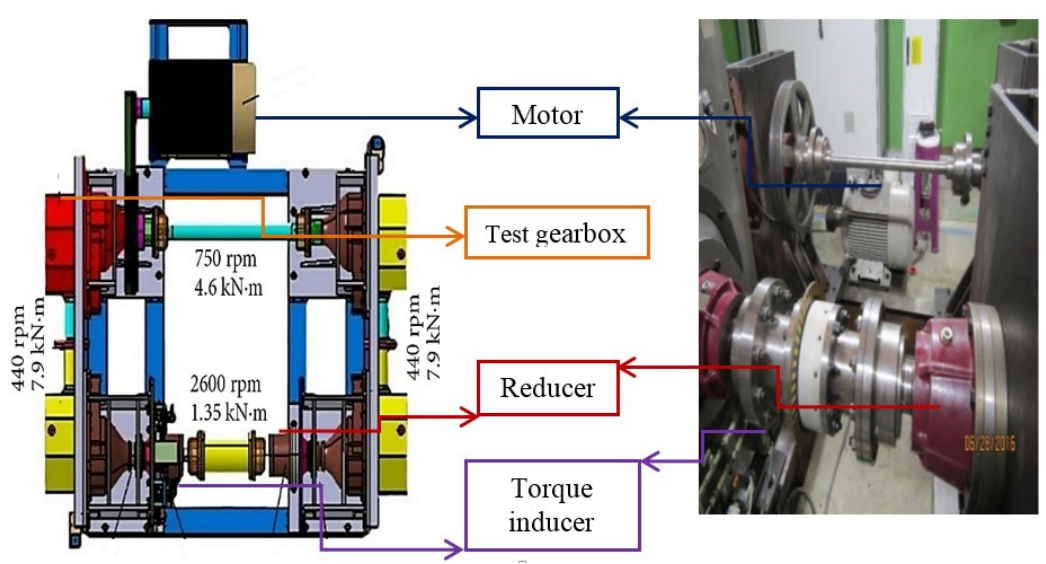

Fig. 1. The schematic and real test rig for high-powered gearboxes

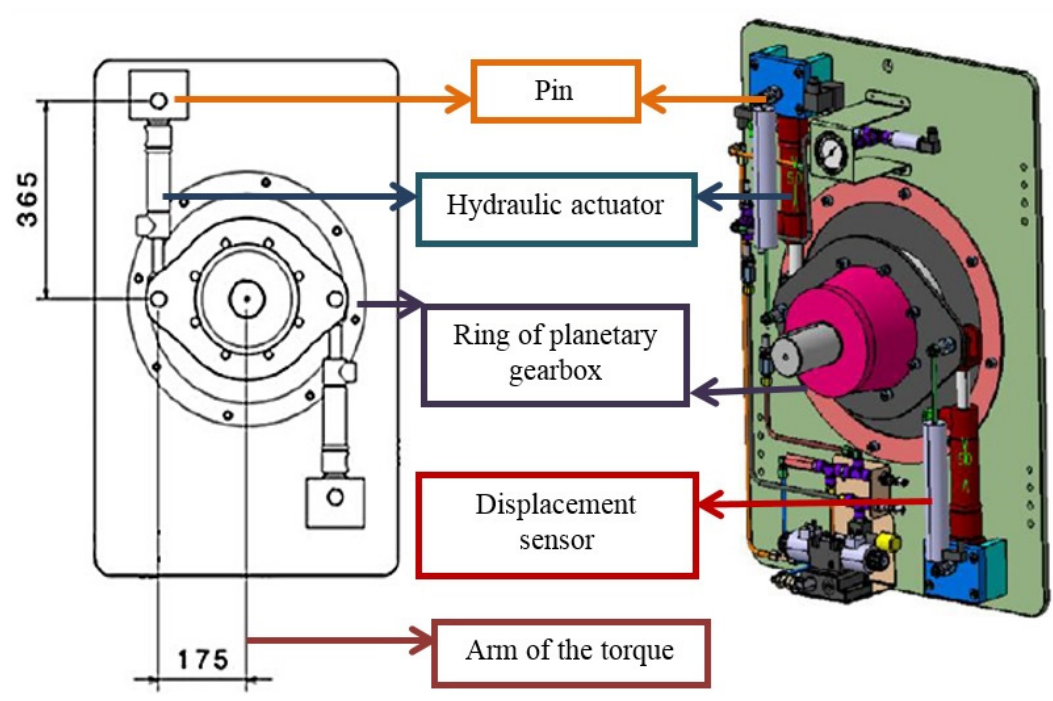

Fig. 2. The schematic and real test rig for high-powered gearboxes

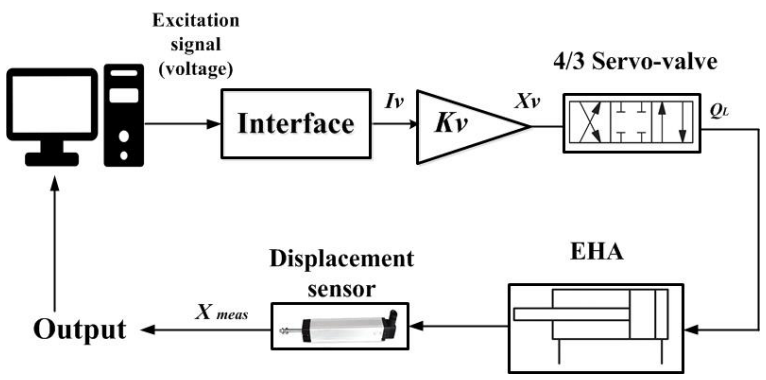

Fig. 3. Data- acquisition procedure

According to Fig. 3, the generated excitation signal in the computer is converted to the current of $I_{v}$. Then, it is multiplied by $K_{v}$, which is the constant gain of the servo valve, to produce $X_{v}$, the signal for displacing the spool of $4 / 3$ servo valve. By displacing the spool, the flow rate of $Q_{L}$ would be changed; thus, the piston would be displaced. Then, this displacement is measured by the displacement sensors that are embedded in the system. The combination of sine signals is the best choice for excitation signal in the cases in which the system would be operated in determined frequencies, and the quality of the collected data in those signals are important. The frequency of the excitation signal should be selected according to the operational frequencies of the system [21]. In this research, the excitation signal is considered as follows:

$$
S_{\text {exc }}=\sum_{i=1}^{n} a_{i} \cos \omega_{i} t_{s},
$$

where $S_{\text {exc }}$ is the excitation signal, $n$ is the number of sine signals that are summed together, $t_{s}$ is the sampling time, $a_{i}$ is the amplitude of $i^{\text {th }}$ sine signals, and $\omega_{i}$ is the frequency of the signals. The gathered input-output signal is depicted in Fig. 4. 

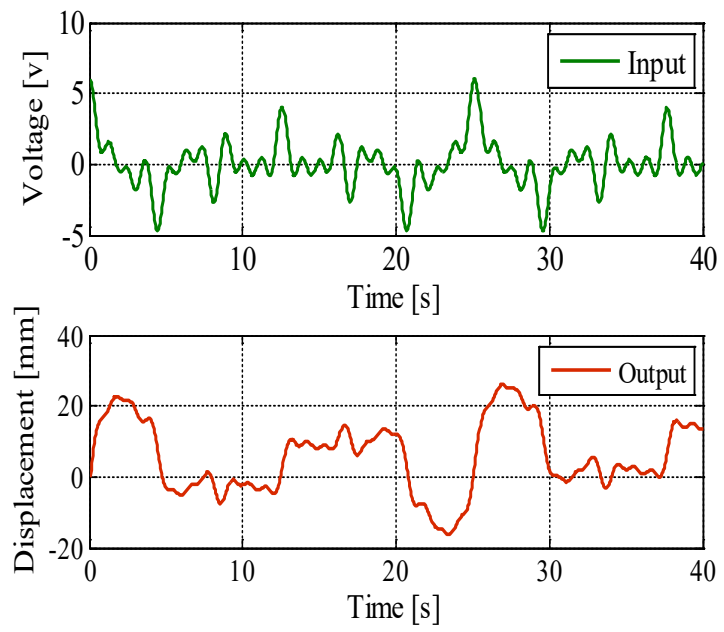

Fig. 4. Input-output data set for training the system

\section{PROBLEM DEFINITION}

For obtaining the model of the actuation system for the torque-applying system, we aim to use a procedure that captures the system dynamics completely and uses no simplifying assumptions and can overcome the existing nonlinearities and uncertainties. Precise modelling is of great importance, as it directly affects the performance of the controller for the actuation system and consequently, the performance of the testing procedure for high-powered gearboxes.

As mentioned, EHA is a single-input singleoutput (SISO) discrete-time nonlinear system. The system can be described using the following relation [23].

$$
x(k+1)=M\left(\begin{array}{l}
u(k), u(k-1), \ldots, u\left(k-n_{a}\right) \\
x(k), x(k-1), \ldots, x\left(k-n_{b}\right)
\end{array}\right) .
$$

This structure provides a nonlinear mapping from input space $\mathbb{R}^{n}$ to output space $\mathbb{R}^{m}$ and defined using $\left[u(k) u(k-1) \ldots u\left(k-n_{a}\right)\right]$ and $\left[u(k) u(k-1) \ldots u\left(k-n_{b}\right)\right]$ vectors. In this relation, $x(k)$ denotes the output of the system at time instant $k$, which is the rod displacement. $\mathrm{M}(.) \in \mathbb{R}$ is a function from $\mathbb{R}^{n} \rightarrow \mathbb{R}^{m}$ and represents the model structure of the system, which is a function of previous inputs and previous outputs of the system. The controller for this plant can be defined as:

$$
u(k)=C\left(\begin{array}{c}
u(k-1), u(k-2), \ldots, u\left(k-n_{c}\right) \\
e_{c}(k+1), e_{c}(k+1), \ldots, e_{c}\left(k-n_{d}\right)
\end{array}\right) .
$$

In Eq. (3), $u(k)$ is the current control signal, which is defined by the use of $\left[u(k-1) u(k-2) \ldots u\left(k-n_{c}\right)\right]$ and $\left[e_{c}(k-1) e_{c}(k-2) \ldots e_{c}\left(k-n_{d}\right)\right]$. $\left[\begin{array}{llll}u(k-1) & u(k-2) & \ldots & u(k-)\end{array}\right]$ denotes the past control inputs, with the $n_{c}$ representing the maximum previous input; while $\left[e_{c}(k-1) e_{c}(k-2) \ldots e_{c}\left(k-n_{d}\right)\right]$ denotes the past errors, with $n_{d}$ representing the maximum past errors. In addition, $C($.$) is a nonlinear function$ representing the controller structure. $e_{c}$ is the tracking error that is defined as:

$$
e_{c}=x(k)-x_{d e s}(k),
$$

where $x_{\text {des }}(k)$ is the desired output.

In order to obtain the model and controller structure practically, some assumptions should be considered as follows:

Assumption 1: The partial derivatives of $M($.$) are$ continuous for $k \in \mathbb{N}$ and $M(.) \in \mathbb{R}$ is a smooth function of $\mathbb{R}^{n} \rightarrow \mathbb{R}^{m}$. This assumption is a general condition for nonlinear systems.

Assumption 2: The model of the system as described in Eq. (2), is generalized Lipchitz; hence a positive constant $C$ exists, so that the $|\Delta x(K+1)| \leq C|| \xi_{k} \|, \xi_{k}=[\Delta x(k), \Delta u(k)]$. This assumption represents the direct influence of the inputs variation on variation rate of the system output. According to this assumption, the outputs of the system are bounded if the inputs are varied in the bounded region [29].

According to the above assumptions, Eq. (2) can be defined by the use of $\phi_{k}$ in the following form:

$$
\begin{aligned}
x(k+1) & =x(k)+\xi_{k}^{T} \phi_{k}= \\
& =x(k)+\Delta x(k) \phi_{1, k}+\Delta u(k) \phi_{2, k} .
\end{aligned}
$$

In Eq. (5) $\phi_{k}$ represents the nonlinear dynamic of the system. The main problem is the estimation of $\phi_{k}$. In this paper, the neural networks are used for approximating this function. A neural network is defined as a system of neurons locating at different layers. With the appropriate selection of the activation functions, bounding the input values to $S_{B} \in \mathbb{R}^{n}$, as well as adjusting the hidden layers number, weights and biases, the $M(x)$, which represents the model of the plant can be defined in the form of:

$$
M(x)=H_{2}{ }^{T} \alpha_{M}\left(H_{1}^{T} x\right)+\varepsilon_{M}(x) .
$$

In the above equation, $H_{1}$ is the matrix representing the weights $\left(\omega_{i 1}\right)$ and biases $\left(b_{i 1}\right)$ of the first hidden layer; while weights $\left(\omega_{i 2}\right)$ and biases $\left(b_{i 2}\right)$ of the second hidden layer are included in $\mathrm{H}_{2}$. Additionally, the vectors of activation functions are denoted by $\alpha_{M}$. Moreover, $\varepsilon_{M}(x)$ is the approximation error by the neural network structure. With the use of a suitable $\mathrm{NN}$ for approximation, which means the appropriate selection of $H_{1}, H_{2}$ and $\alpha_{M}$, it is necessary 
to obtain $M(x)$ so that the approximation error $\left(\varepsilon_{M}(x)\right)$ becomes less or equal to the acceptable error:

$$
\varepsilon_{M}(x) \leq \varepsilon_{M R} \text { for all samples. }
$$

$\varepsilon_{M R}$ is the acceptable error, which is defend by the problem dynamics and proposed application. It should be noted that the computational costs would be increased for the lower amount of this value. After that, the appropriate estimation for the model is achieved, all the biases and weights are considered fixed, and their learning rate set to zero $\left(\omega_{i 1}, b_{i 1}, \omega_{i 2}\right.$ and $b_{i 2}$, = const., hence, $H_{1}, H_{2}$ and $\alpha_{M}=$ const.). Therefore, that the fixed model of the system is available for the further uses in the controller.

The schematic of the proposed procedure is depicted in Fig. 5. As is obvious, two networks are used in the proposed process; one represents the model of the EHA while the other is used as a controller. The neuron inputs (current and previous inputs; in this research past two inputs are used) are multiplied by the corresponding weights, then the resultant products are summed together their summation with biases are fed into a transfer function. and the output is generated as follows:

$$
x=M\left(\sum_{i=0}^{i=1}\left(H_{i} H_{i+1}+\varepsilon_{i}\right)\right) .
$$

The adaption of $\mathrm{NN}$ weights are based on Levenberg-Marquandt algorithm. Another neural network structure is used as the controller of the EHA.
$C(x)$ can be defined as the following structure, which includes two hidden layers.

$$
C(x)=H_{4}^{T} \alpha_{C}\left(H_{3}^{T} x\right)+e_{C}(x) .
$$

In Eq. (8), $H_{3}$ is the matrix representing the weights $\left(\omega_{i 3}\right)$ and biases $\left(b_{i 3}\right)$ of the first hidden layer in the controller structure. Weights $\left(\omega_{i 4}\right)$ and biases $\left(b_{i 4}\right)$ of the second hidden layer in the controller design are included in $H_{4}$. Additionally, the vector of activation functions is denoted by $\alpha_{C}$. By using suitable NNs for the controller structure, which means the appropriate selection of $H_{3}, H_{4}$ and $\alpha_{C}$, it is necessary to obtain $C(x)$ so that the controller steady-state error $\left(e_{C}(x)\right)$ is located in the acceptable range:

$$
\varepsilon_{C}(x) \leq \varepsilon_{C R} \text { for all samples. }
$$

In summary, the outputs of the first neural network (controller section) is the controller command for the model, which is obtained through the second neural network (model section). In this research, the modelling is conducted offline, meaning that first the data is collected and then, it is transferred to the personal computer (PC) for processing and obtaining the model. Once the model is obtained, there is no need for calculation in further uses. Because the actuation system is responsible for running the whole system, controlling the displacement of the hydraulic actuator is very important. Different parameters, including the type of input/output signals, the number of inputs/outputs and the appropriate controlling

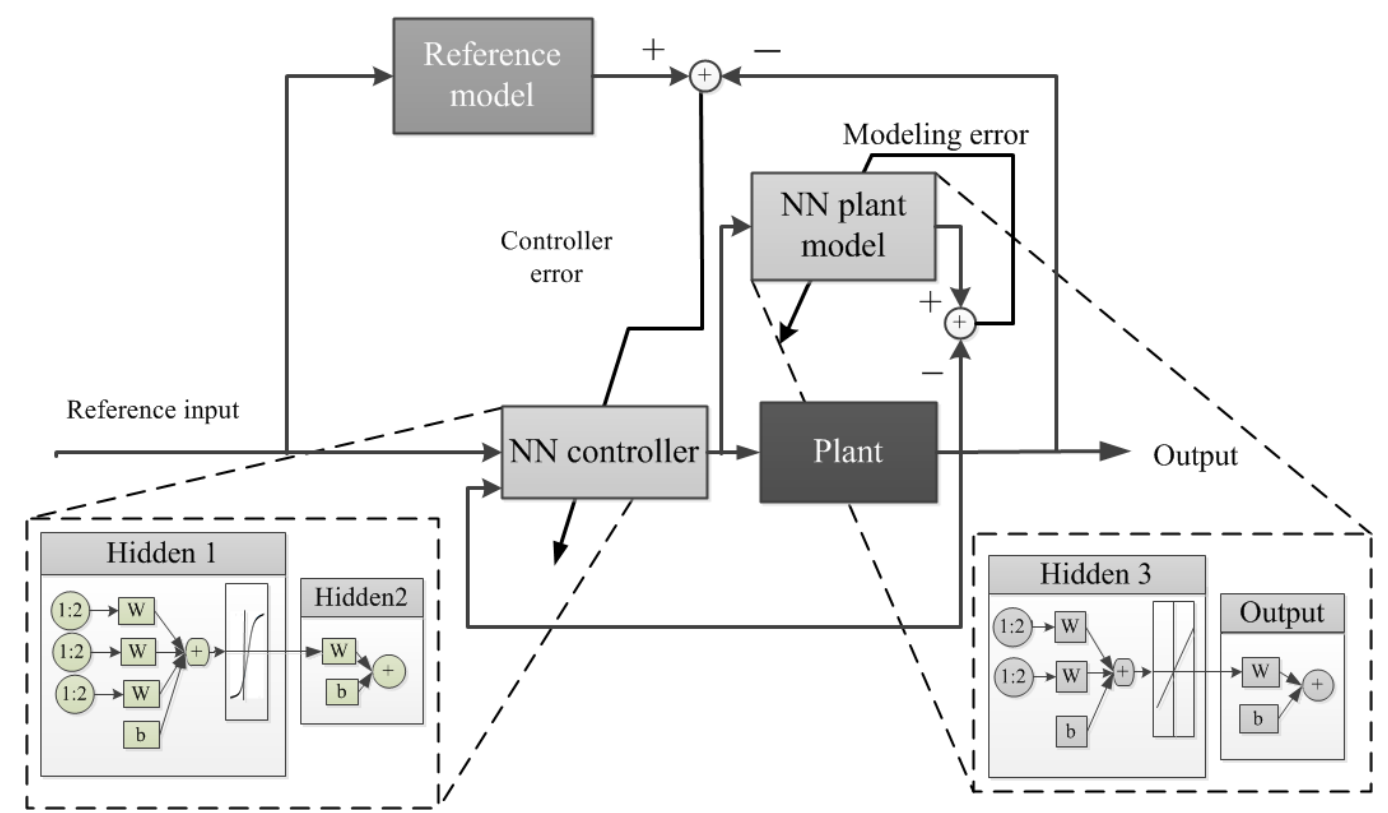

Fig. 5. The proposed procedure for modelling and controller design 
algorithm, should be considered in the control scheme. In the desired test rig, hydraulic actuators are in displacement control mode, which means that the command signal that is fed to the servo valve is based on the difference between the desired displacement and measured displacement by the sensors. The steps taken for modelling and controlling the system are depicted in Fig. 6.

\subsection{Model Estimation and Validation}

The criterion for selecting the model with the best quality is the root mean square error (RSME) criteria. This criterion is the most-widely used measure of difference between the predicted values through the obtained model and observed values through the embedded sensors. This criterion is defined as follows:

$$
R S M E=\sqrt{\left(\sum_{i=1}^{n}\|x(k)-\widehat{x}(k)\|_{2}\right) / n,}
$$

where, $x(k)$ and $\widehat{x}(k)$ are the real and predicted outputs of the system and $n$ is the sample number. This term is always between 0 and 1; smaller values indicate the high quality of the predicted values. In addition, the error mean and std (standard deviation) are also checked. The error mean is the average of the errors; while std provides an indication of how far the responses deviate from the mean.

\section{RESULTS AND DISCUSSION}

In this section, the obtained results are provided and discussed. The results are represented in two sections; the first section is dedicated to the modelling, and the second section is devoted to the controlling results.

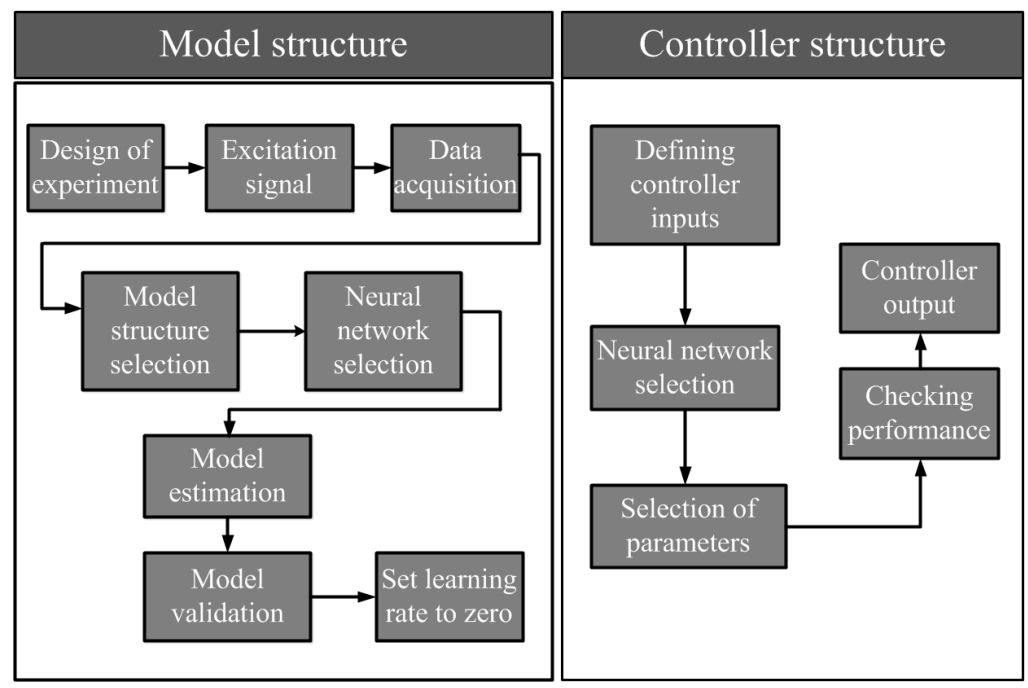

Fig. 6. Neural-Network based model and controller

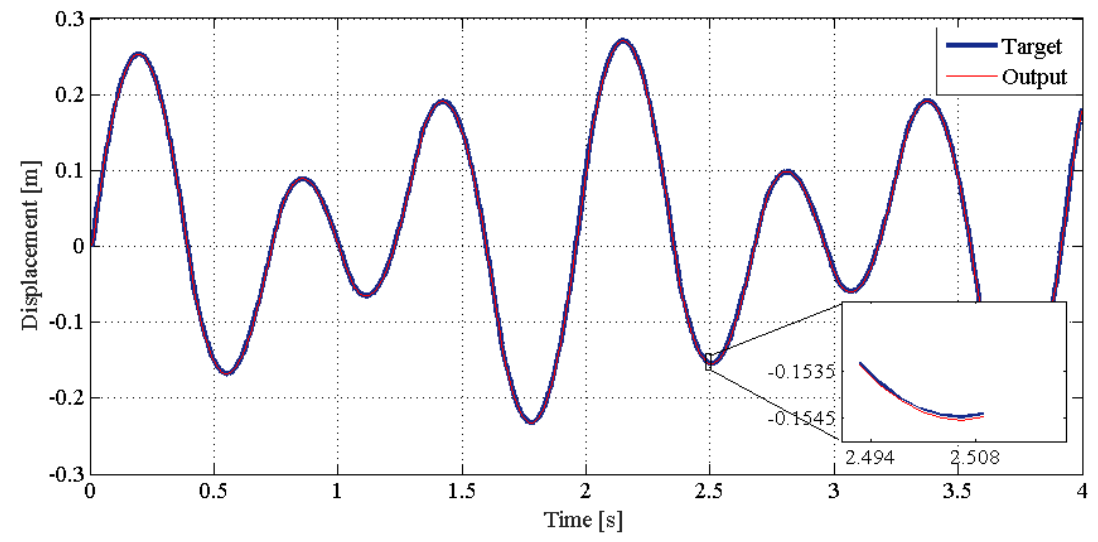

Fig. 7. Modelling results: The blue line: Target values and red line: Output values 


\subsection{Modelling Results}

The results of modelling the system are depicted in Fig. 7. As shown in this figure, the estimated model can capture the dynamic of the system with an acceptable error. A magnified portion of the figure between the $2.49 \mathrm{~s}$ and $2.51 \mathrm{~s}$ shows the small deviation between the real and estimated models. In addition, the RSME, mean and std (standard deviation) of error are shown in Fig. 8. According to Fig. 8, the RSME of the error is equal to 0.0055726 , which confirms the validity of the estimated model. Since it is close to zero, it clarifies the quality of the model. Additionally, std is equal to 0.0005574 , and its low value indicates the closeness of the values to the mean value.
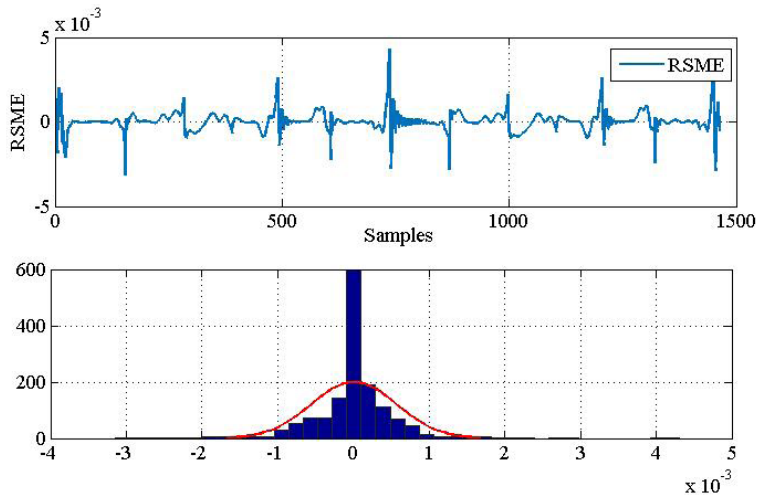

Fig. 8. RSME and error properties for modelling results; a) RSME diagram (RSME=0.00055726), and b) Error mean and standard deviation diagram (error mean $=7.0024 \mathrm{e}-0.6, \mathrm{std}=0.0005574$ )

\subsection{Controller Result}

As mentioned, a neural network controller was utilized for controlling the position of the hydraulic actuator. The performance of the designed controller in different conditions is investigated in the following sections. The desired reference is considered in the workspace of the hydraulic actuator, in which the model was trained. Two constant and variable step references are considered as the references for the system and the performance of the system in two conditions, normal condition and in the presence of the disturbance, is studied. The results are provided in Figs. 9 to 12. As can be seen in Fig. 9 for the constant reference in normal condition, the controller output tracks the reference output in less than $0.25 \mathrm{~s}$. According to this figure, no overshoot is seen in the controller output; in addition, it tracks the reference without any steady-state error. For the variable step reference, the controllers track the reference without any steady-state error. The settling time is different

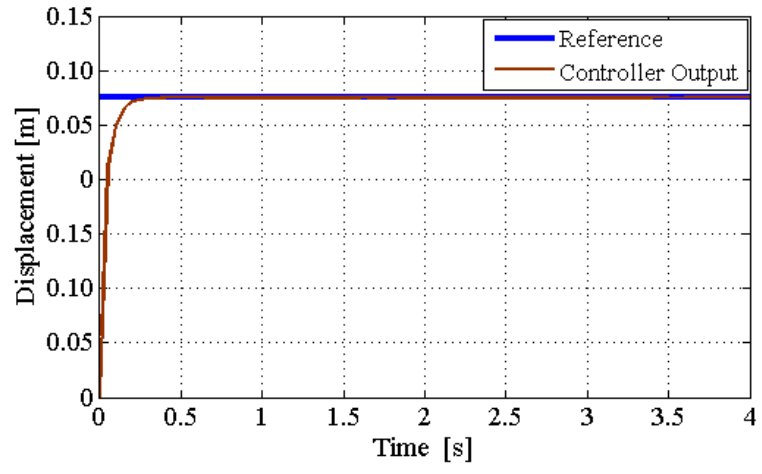

Fig. 9. Controller performance for constant reference

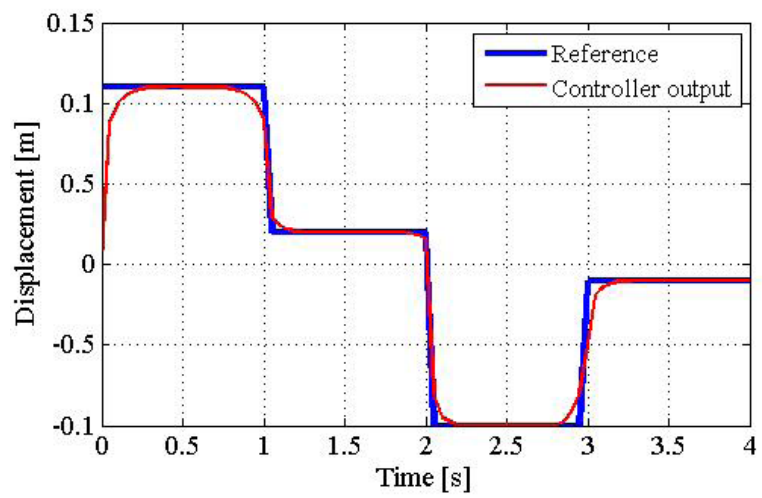

Fig. 10. Controller performance for variable reference

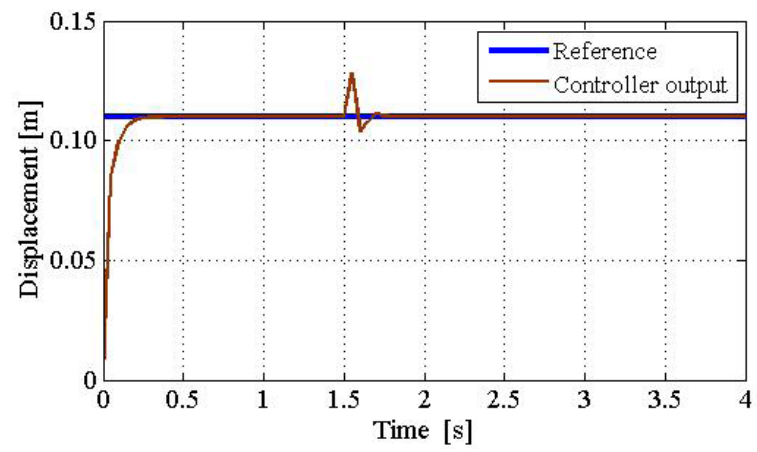

Fig. 11. Controller performance for constant reference in the presence of disturbance

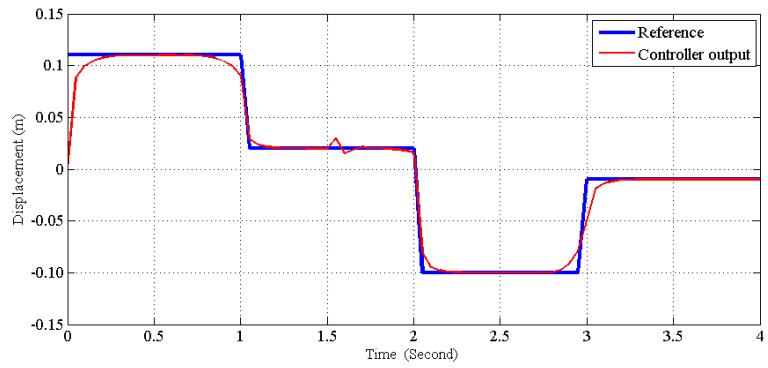

Fig. 12. Performance of the controller for variable reference in the presence of disturbance 
for different steps. After a maximum of $0.3 \mathrm{~s}$, the controller output tracks the determined variable step.

The performance of the controller in the presence of the disturbance are presented in Figs. 11 and 12 for constant reference and variable references, respectively. The disturbance in the form of

$$
\begin{aligned}
\text { dist } & =0.04(t>1.50)(t \leq 1.55)- \\
& -0.02(t>1.55)(t \leq 1.7),
\end{aligned}
$$

was applied to the system in the time duration of $1.50 \mathrm{~s}$ and $1.7 \mathrm{~s}$. According to Fig. 11 and Fig. 12, the controller can damp the disturbance well and the systems tracks the reference after that the disturbance is applied for a short period.

\subsection{Comparison with PID Control Scheme}

In order to highlight the superiority of the obtained results from the NN controller, we aimed to provide a comparison with conventional PID results. This controller is also considered to be a model-free controller. The control goal was to track a constant reference. For this purpose, the PID controller is defined as:

$$
u(t)=K_{p} e(t)+K_{i} \int_{0}^{t} e\left(t^{\prime}\right) d t^{\prime}+K_{d} \frac{d e(t)}{d t} .
$$

In which, $K_{p}, K_{i}$ and $K_{d}$ denote the proportional, integral, and derivative coefficients, respectively. Additionally, $e(t)$ represents the error, which is defined as:

$$
e(t)=x_{d}(t)-x(t) .
$$

where $x_{d}(t)$ is the desired output and $x(t)$ is the output, obtained from the controlled plant. The aim was to provide the minimum error by the use of the controller. The PID control scheme is depicted in Fig. 13. The result of implementing PID controller along with NN controller is plotted in Fig. 14 for tracking the constant reference. According to this figure, the $\mathrm{NN}$ controller provides faster convergence with the reference input. In both controllers, no steady-state error is seen; however, the settling time for the PID controller is much bigger compared to the $\mathrm{NN}$ controller.

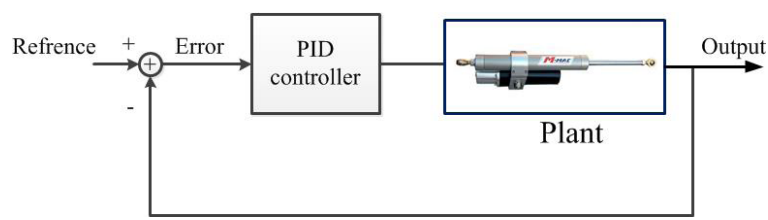

Fig. 13. The PID control scheme

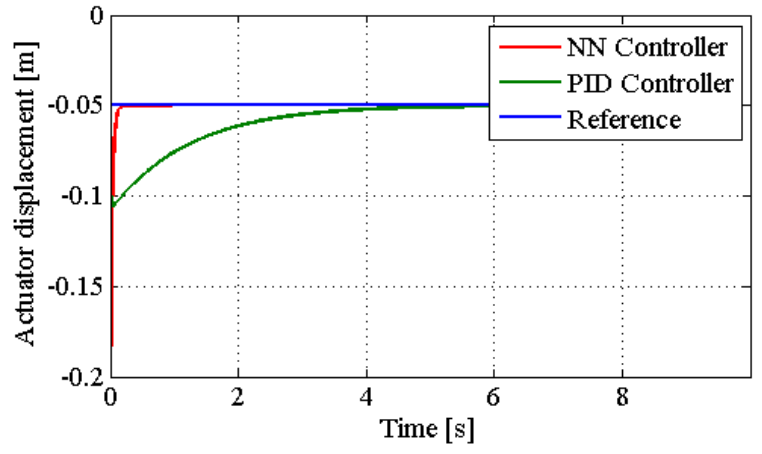

Fig. 14. Comparison between the results of NN controller and PID controller

For the NN controller, the settling time is approximately $0.25 \mathrm{~s}$; while for the PID controller, this value is $4.35 \mathrm{~s}$. In both controllers, no overshoot was observed. Therefore, it can be concluded that the NN controller performs better.

\section{CONCLUSION}

Different methods were used for ensuring the safety and performance of the high-powered gearboxes that are mostly used in aeronautic industries. The usage of real-sized test rigs for evaluating the performance of system components is known to be one of the reliable methods. In order to provide variable torque and speed, torque applying systems are required. In this paper, we investigated the hydraulically-driven actuation system for the torque applying system of the test rig that was designed and fabricated in Sharif University of Technology branch of ACECR. First, identification and data-driven modelling of the hydraulically-actuated torque applying system of a mechanically closed-loop test rig were conducted by neural networks. The advantage of the proposed scheme is capturing the whole system dynamic, which was problematic due to the existing nonlinearities in the dynamic of the EHA, In addition, the modelling was conducted without any simplifying assumptions. Therefore, the precise model of the EHA was obtained without performing time-consuming mathematical calculations. After that, the model was obtained, a neural network controller was designed to track the desired output; the parameters of this network was adjusted so that the tracking error becomes very small. According to the obtained results, the system was modelled with the RMSE of 0.00055726 and std of 0.0005574 , which indicates the good quality of the model. For the controller, it was obvious that the controller output reached to the reference output in approximately $0.25 \mathrm{~s}$, without any overshoot and 
steady-state error. In addition, the controller tracked the variable reference very well. For the case in which the disturbance was presented, the controller was able to deal with the applied disturbances and followed the reference after $0.13 \mathrm{~s}$. In summary, a good tracking feature of the controller was obtained in different conditions; in addition, good disturbance rejection feature was observed. It should be mentioned that the use of two neural networks increases the computational cost of the system. In order to solve this problem, in this paper, it was proposed to set the learning factors of the model section to zero. Finally, to highlight the superior characteristics of the proposed data-driven algorithm, the results of this paper was compared with the results of the conventional PID controller. In general, the proposed Neural Network modelling, and the Neural Network controller provide acceptable results, in which the system dynamics include uncertainty and non-modelled nonlinearity.

\section{REFERENCES}

[1] Averous, N.R., Stieneker, M., Kock, S., Andrei, C., Helmedag, A., De Doncker, R.W., Hameyer, K., Jacobs, G., Monti, A. (2017). Development of a $4 \mathrm{MW}$ full-size wind-turbine test bench. IEEE Journal of Emerging and Selected Topics in Power Electronics, vol. 5, no. 2, p. 600-609, D0l:10.1109/jestpe.2017.2667399.

[2] Zhou, L., Duan, F., Corsar, M., Elasha, F., Mba, D. (2019). A study on helicopter main gearbox planetary bearing fault diagnosis. Applied Acoustics, vol. 147, p. 4-14, D0l:10.1016/j. apacoust.2017.12.004.

[3] Åkerblom, M. (2008). Gearbox Noise: Correlation with Transmission Error and Influence of Bearing Preload, $\mathrm{PhD}$ thesis, KTH, School of Industrial Engineering and Management, Stockholm.

[4] Arun, A.P., Senthil Kumar, A.P., Giriraj, B., Rahaman, A.F. (2014). Gear test rig - A review. International Journal of Mechanical \& Mechatronics Engineering, vol. 14, no. 5, p. 1626.

[5] Mihailidis, A., Nerantzis, I. (2009). A new system for testing gears under variable torque and speed. Recent Patents on Mechanical Engineering, vol. 2, no. 3, p. 179-192, D0l:10.21 74/2212797610902030179.

[6] Palermo, A., Anthonis, J., Mundo, D., Desmet, W. (2014). A novel gear test rig with adjustable shaft compliance and misalignments, Part I: Design. Advances in Condition Monitoring of Machinery in Non-Stationary Operations, Lecture Notes in Mechanical Engineering, p. 497-506, DOI:10.1007/978-3-642-39348-8_43.

[7] Mozafari, S., Rezazadeh. M., Takloo, S.D., Mardani, M. (2017). Design of a mechanically closed-loop test rig for testing aviation industry's gearboxes. Aviation, vol. 21, no. 4, pp. 132142, Dol:10.3846/16487788.2017.1415225.

[8] Mardani, M. (2018). Mechanically Closed Loop Gearbox Test Rig Controller. Control Engineering Letters, vol. 3, no. 1, p. 1226.
[9] Takloo, S.D., Mozafari, S., Rezazadehmohamadi, M., \& Mardani, M. (2017). Fractional order PID control mechanism for helicopter gearbox test control with internal and external disturbance. Bulletin de la Société Royale des Sciences de Liège, vol. 86, p. 27-138, D0l:10.25518/0037-9565.6619.

[10] Lee, W., Chung, W.K. (2019). Disturbance-observer-based compliance control of electro-hydraulic actuators with backdrivability. IEEE Robotics and Automation Letters, vol. 4, no. 2, p. 1722-1729, D0I:10.1109/LRA.2019.2897178.

[11] Lee, W., Kim, M.J., Chung, W.K. (2017). Disturbanceobserver-based PD control of electro-hydrostatically actuated flexible joint robots. IEEE/RSJ International Conference on Intelligent Robots and Systems, p. 2821-2828, D0I:10.1109/ IROS.2017.8206113.

[12] Detiček, E., Kastrevc, M. (2016). Design of Lyapunov based nonlinear position control of electrohydraulic servo systems. Strojniški vestnik - Journal of Mechanical Engineering, vol. 62, no. 3, p. 163-170, Dol:10.5545/sv-jme.2015.2921.

[13] Maslov, D.A., Merkuryev, I.V. (2018). Increase in the accuracy of the parameters identification for a vibrating ring microgyroscope operating in the forced oscillation mode with nonlinearity taken into account. Russian Journal of Nonlinear Dynamics, vol. 14, no. 3, pp. 377-386, D0l:10.20537/ nd180308.

[14] Vasiukova, O.E., Klimina, L. A. (2018). Modelling of selfoscillations of a controlled pendulum with respect to a friction torque depending on a normal reaction in a joint. Russian Journal of Nonlinear Dynamics, vol. 14, no. 1, p. 33-44, DOI:10.20537/nd1801003.

[15] Ledezma Pérez, J.A., De Pieri, E.R., De Negri, V.J. (2018). Force control of hydraulic actuators using additional hydraulic compliance. Strojniški vestnik - Journal of Mechanical Engineering, vol. 64, no. 10, p. 579-58, Dol:10.5545/svjme.2018.5339.

[16] Won, D., Kim, W., Tomizuka, M. (2017). High-gain-observerbased integral sliding mode control for position tracking of electrohydraulic servo systems. IEEE/ASME Transactions on Mechatronics, vol. 22, no. 6, p. 2695-2704, D0l:10.1109/ TMECH.2017.2764110.

[17] Huang, Y., Pool, D. M., Stroosma, O., Chu, Q. (2019). Longstroke hydraulic robot motion control with incremental nonlinear dynamic inversion. IEEE/ASME Transactions on Mechatronics, vol. 24, no. 1, p. 304-314, D0l:10.1109/ TMECH.2019.2891358.

[18] Guan, C., Pan, S. (2008). Nonlinear adaptive robust control of single-rod electro-hydraulic actuator with unknown nonlinear parameters. IEEE Transactions on Control Systems Technology, vol. 16, no. 3, p. 434-445, D0l:10.1109/ TCST.2007.908195.

[19] Wang, S., Xu, Q., Lin, R., Yang, M., Zheng, W., Wang, Z. (2017). Feedback linearization control for electro-hydraulic servo system based on nonlinear disturbance observer. 36th Chinese Control Conference, p. 4940-4945, D0l:10.23919/ Chicc.2017.8028135.

[20] Yao, J., Yang, G., Jiao, Z. (2015). High dynamic feedback linearization control of hydraulic actuators with backstepping. Proceedings of the Institution of Mechanical Engineers, Part l: 
Journal of Systems and Control Engineering, vol. 229, no. 8, p. 728-737, DOl:10.1177/0959651815581555.

[21] Detiček, E., Župerl, U. (2011). An intelligent electrohydraulic servo drive positioning. Strojniški vestnik - Journal of Mechanical Engineering, vol. 57, no. 5, p. 394-404, DOI:10.5545/sv-jme.2010.081.

[22] Yao, J., Jiao, Z., Ma, D. (2015). A practical nonlinear adaptive control of hydraulic servomechanisms with periodic-like disturbances. IEEE/ASME Transactions on Mechatronics, vol. 20, no. 6, pp. 2752-2760, D0l:10.1109/ TMECH.2015.2409893.

[23] Xu, Y., Bideaux, E., Thomasset, D. (2013). Robustness study on the model-free control and the control with restricted model of a high performance electro-hydraulic system. 13th Scandinavian International Conference on Fluid Power, no. 092, p. 523-530, D0I:10.3384/ecp1392a52.

[24] Xu, Y. (2013). Modelling and Control of a High Performance Electro-Hydraulic Test Bench, PhD thesis, University of Lyon, Institut National des Sciences Appliqués, Lyon.

[25] Yao, Z., Yao, J., Sun, W. (2018). Adaptive RISE control of hydraulic systems with multilayer neural-networks. IEEE
Transactions on Industrial Electronics, vol. 66, no. 11, p. 8638-8647, DOI:10.1109/TIE.2018.2886773.

[26] Hegedüs, F., Bécsi, T., Aradi, S., Gáspár, P. (2019). Motion planning for highly automated road vehicles with a hybrid approach using nonlinear optimization and artificial neural networks. Strojniški vestnik - Journal of Mechanical Engineering, vol. 65, no. 3, p. 148-160, D0l:10.5545/svjme.2018.5802.

[27] dos Santos, J.D.B., Bessa, W. M. (2018). Intelligent control for accurate position tracking of electrohydraulic actuators. Electronics Letters, vol. 55, no. 2, p. 78-80, D0l:10.1049/ el.2018.7218.

[28] Parvaresh, A., Mardani, M. (2019). Model predictive control of a hydraulic actuator in torque applying system of a mechanically closed-loop test rig for the helicopter gearbox. Aviation, vol. 23, no. 4, p. 143-153, Dol:10.3846/ aviation.2019.11869.

[29] Hou, Z., Jin, S. (2010). A novel data-driven control approach for a class of discrete-time nonlinear systems. IEEE Transactions on Control Systems Technology, vol. 19, no. 6, p. 1549-1558, DOI:10.1109/TCST.2010.2093136. 